\title{
Age related metabolic modifications \\ in the migraine brain
}

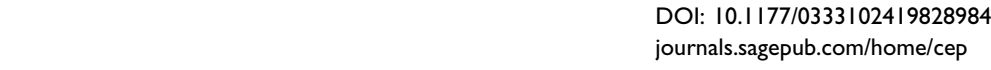

\author{
Marco Lisicki 1,* (D, Kevin D'Ostilio ${ }^{1, *}$, Gianluca Coppola² ${ }^{(D}$, \\ Vincenzo Parisi", Alain Maertens de Noordhout', \\ Delphine Magis' ${ }^{\prime}$, Jean Schoenen', Felix Scholtes ${ }^{3,4, \#}$ and \\ Jan Versijpt ${ }^{5, \#}$
}

\begin{abstract}
Purpose: The aim of this study was to evaluate the possibility that migraine patients exhibit specific age-related metabolic changes in the brain, which occur regardless of disease duration or the frequency of attacks.

Methods: We analysed the relation between brain glucose $\left({ }^{18} \mathrm{~F}\right.$-fluorodeoxyglucose) uptake and age in healthy volunteers $(n=20)$ and episodic migraine patients $(n=19)$. In the latter, we additionally compared the correlation between ${ }^{18} \mathrm{~F}$-fluorodeoxyglucose uptake and disease duration and monthly migraine days.

Results: In contrast to controls, in migraine patients advancing age was positively correlated to increased metabolism in the brainstem (especially the posterior pons), hippocampus, fusiform gyrus and parahippocampus. Conversely, no significant correlations between cerebral metabolism and disease duration or migraine days were observed.

Conclusions: Findings of this cross-sectional study show that episodic migraine patients exhibit specific metabolic brain modifications while ageing. As such, age is correlated with metabolic changes in key regions of the brain previously associated with migraine's pathophysiology to a better extent than disease duration or the number of monthly migraine days. More than the repeated headache attacks, the continuous interaction with the environment seemingly models the brain of migraine sufferers in an adaptive manner. A positive control (e.g. chronic pain) is missing in this study and therefore findings cannot be proven to be migraine-specific.
\end{abstract}

\section{Keywords}

Positron emission tomography, brain metabolism, ageing, allostasis

Date received: 12 October 2018; revised: 17 December 2018; accepted: 15 January 2019

\section{Introduction}

One in every five women and one in every 15 men will suffer from migraine at some point of their life (1). Despite often being neglected, migraine entails a substantial disability at the personal level, and a tremendous burden at the level of society $(2,3)$. More than a recurrent pain syndrome, migraine constitutes a brain disease $(4,5)$. A glance at migraine prevalence curves is all it takes to realize that this condition is strongly affected by the ageing process $(6,7)$. Although hormonal influences are non-neglectable, they are not sufficient to explain the course of the disease in all patients. Indeed, it is not uncommon for migraineurs to have a history of painless clinical manifestations (e.g. somnambulism, cyclic vomiting, vertigo) that occurred early during childhood, years before puberty, and also years before the diagnosis of migraine was actually

\footnotetext{
'Headache Research Unit, University Department of Neurology CHR, Citadelle Hospital, University of Liège, Liège, Belgium

${ }^{2}$ IRCCS - Fondazione Bietti, Research Unit of Neurophysiology of Vision and Neurophthalmology, Rome, Italy

${ }^{3}$ Department of Neurosurgery, University of Liège, Liège, Belgium

${ }^{4}$ Department of Neuroanatomy, University of Liège, Liège, Belgium

${ }^{5}$ Vrije Universiteit Brussel (VUB), Universitair Ziekenhuis Brussel

(UZ Brussel), Department of Neurology, Brussels, Belgium

*These authors contributed equally to this work.

\#These authors are shared last authors.

\section{Corresponding author:}

Jan Versijpt, Vrije Universiteit Brussel (VUB), Universitair Ziekenhuis Brussel (UZ Brussel), Department of Neurology, Laarbeeklaan I0I, 1090 Brussels, Belgium.

Email: Jan.versijpt@uzbrussel.be
} 
established (8). Further in life, towards old age, migraine tends to spontaneously remit, a phenomenon perhaps less linked to the menopause than previously thought (9).

Recently, in line with theoretical models (4), results from an MRI study have suggested that the migraine brain undergoes age-related structural modifications regardless of the duration of the disease or the attack frequency (10). This phenomenon has never been analysed from a metabolic perspective. The aim of the present study was to evaluate the correlation between cerebral glucose $\left({ }^{18} \mathrm{~F}\right.$-fluorodeoxyglucose $)$ uptake and age in healthy volunteers and migraine patients. In addition, in order to separately evaluate the influence of age, disease duration, and migraine days on brain metabolism, we performed multivariate regressions for these variables in a group of episodic migraine without aura patients.

\section{Subjects and methods}

\section{Subjects}

Forty-one subjects participated in this study: 20 healthy volunteers and 21 episodic migraine patients (migraine without aura patients diagnosed according to The International Classification of Headache Disorders 3rd edition (beta version) of the International Headache Society) (11) recruited by posting advertisements on the University web page or via the headache clinic. Except for migraine, subjects were devoid of any neurologic or systemic disease. Healthy volunteers did not report having any first-degree relative suffering from recurrent headache of any type. Patients were not under any migraine preventive treatment for at least 30 days preceding imaging. Only the scans of migraine patients performed during the interictal period (at least 48 hours after their last headache episode, and at least 48 hours before the next one) were included in the analyses. For this reason, scans from two patients had to be excluded after headache diary examination.

\section{Compliance with ethical standards}

Ethical approval was granted by the Institution's ethics committee (Centre Hospitalier Régional de la Citadelle, Liège, Belgium - protocol n ${ }^{\circ} 1422$ ). The study was conducted in accordance with the ethical standards of the institutional research committee and with the 1964 Helsinki declaration and its later amendments or comparable ethical standards. Informed consent was obtained from all individual participants included in the study. This project forms part of the EUROHEADPAIN project, FP7 n 602633 .

\section{FDG-PET}

PET acquisitions were made in the Nuclear Medicine Department of the Centre Hospitalier Universitaire (CHU) Sart Tilman, Liège, Belgium, using a Gemini TF PET/computed tomography (CT) scanner (Philips, Eindhoven, The Netherlands). Resting cerebral metabolism was studied 30 minutes after intravenous injection of $150 \mathrm{MBq}{ }^{18} \mathrm{~F}$-fluorodeoxyglucose (FDG). Blood glucose level was lower than $110 \mathrm{mg} / \mathrm{dl}$ in all participants. Subjects were injected and scanned in a dark room with minimal environmental noise. Images were reconstructed using an iterative list mode time-of-flight algorithm. Corrections for attenuation, dead-time, random and scatter events were applied.

\section{Image analyses}

PET scans were analysed using Statistical Parametric Mapping version 12 (SPM12, Wellcome Trust Centre for Neuroimaging, http://www.fil.ion.ucl.ac.uk/spm) implemented in MATLAB 7.4.0 (MathWorks Inc, Sherborn, MA, USA). Images were first manually reoriented and then spatially normalised into a standard stereotactic space using an MNI PET template (Montreal Neurological Institute) and smoothed using an $8 \mathrm{~mm}$ full-width-half-maximum isotropic kernel. We performed global normalisation by applying proportional scaling. In order to evaluate the brain metabolic modifications associated with age, we identified voxels in which age was correlated with resting ${ }^{18} \mathrm{FDG}$ uptake. Positive and negative correlations with age were explored. We also separately performed voxel-wise multiple regression analyses to test the effect of age, disease duration, and migraine days on brain metabolism, when acting as either variables of interest or nuisance variables. Finally, we performed t-tests (one-sided t-tests for positive and negative contrasts) to compare resting ${ }^{18} \mathrm{FDG}$ uptake in migraine patients and healthy controls with and without controlling for age. Unless otherwise clarified, only regions surviving a peak or cluster $\mathrm{pFWE}<0.05$ correction at a whole brain level are reported in the results section. The statistical significance threshold was set at $p<0.001$ (uncorrected) for exploratory and display purposes. No constraints regarding the minimum extent threshold (number of voxels) for detecting clusters were applied. Brain regions were identified using the Neuromorphometrics atlas (Somerville, MA, USA) implemented in SPM12. 


\section{Results}

\section{Participants' characteristics}

Participants' characteristics are summarized in Table 1. No differences in age means $(\mathrm{t}=0.415 ; p=0.68)$, age variances $(\mathrm{F}=1.631 ; p=0.30)$, or gender proportions $\left(\chi^{2}=0.008 ; p=0.93\right)$ were observed between migraine patients and controls. The age range in both groups extended over the migraine prevalence peak (Figure 1).

\section{Age-metabolism correlations and correlations with other clinical variables in migraine}

In correlation with age, migraine patients exhibited significantly augmented FDG uptake in the brainstem (posterior pons) $(-4,-38,-38 ; \mathrm{T}=7.42$; peak level $\mathrm{pFWE}=0.045$ ), and bilaterally in two large, almost symmetrical, clusters mainly comprising the parahippocampal and fusiform gyrus, and the hippocampus, with

Table I. Characteristics of the participants. Migraine days refers to the monthly days with migraine headache assessed through inspection of patients' calendars. Disease duration is expressed in years.

\begin{tabular}{lll}
\hline & $\begin{array}{l}\text { Healthy } \\
\text { volunteers }\end{array}$ & $\begin{array}{l}\text { Episodic } \\
\text { migraine }\end{array}$ \\
\hline $\mathrm{n}$ & 20 & 19 \\
Age (mean \pm SD) & $36.10 \pm 1 \mathrm{I} .4$ & $34.37 \pm 14.6$ \\
Age range (min-max) & $21-59$ & $20-63$ \\
Female (\%) & 75.0 & 79.0 \\
Migraine days (mean $\pm S D)$ & & $4.6 \pm 2.5$ \\
Disease duration $(m e a n \pm S D)$ & & $15.2 \pm 11.1$ \\
\hline
\end{tabular}

SD: standard deviation.

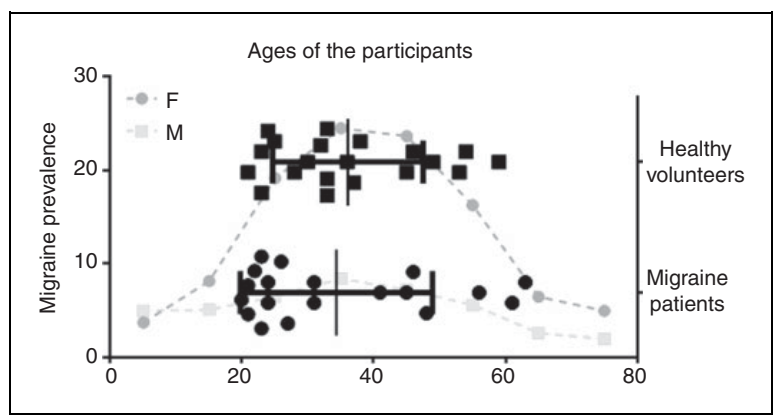

Figure I. Ages of the participants in each group plotted over estimated migraine prevalence curves (left y axis corresponds to migraine prevalence (\%)) separated by sex $(7,29)$. Mean and standard deviations are also depicted. some voxels extending over the thalamus (right: 32 , $-20,-22 ; \mathrm{T}=6.65$; cluster level $\mathrm{pFWE}<0.001$; left $-26,-34,-4 ; \mathrm{T}=6.41$; cluster level $\mathrm{pFWE}<0.001$ ) (Figure 2). The strongest negative correlation (right temporo-parietal junction: $48,-48,14 ; \mathrm{T}=7.19$; peak level $\mathrm{pFWE}=0.062$ ), fell slightly above the statistical significance threshold chosen a priori (i.e. $\mathrm{pFWE}=0.05)$.

Conversely, no significant correlation was observed between age and FDG uptake in healthy volunteers.

No significant correlations, whether positive or negative, were observed between disease duration or migraine days and FDG uptake. Correlations at a more permissive statistical threshold $(p<0.001$ uncorrected) are depicted in Figure 3 for comparison purposes. The region in the posterior pons positively correlated with age in migraine patients remained significant after controlling for disease duration $(5 \mathrm{~mm}$ sphere small volume correction at $-4,-38,-38$; $\mathrm{T}=5.79$; peak level $\mathrm{pFWE}<0.001$ ) or migraine days $(5 \mathrm{~mm}$ sphere small volume correction at $-4,-38$, $-38 ; \mathrm{T}=7.22$; peak level pFWE $<0.001$ ) (Figure 4).

\section{Between group comparisons}

The comparison between healthy volunteers and migraine patients revealed a cluster of significantly reduced metabolism in migraineurs located in the right post-central gyrus extending towards the subjacent white matter, both with $(34,-42,52 ; \mathrm{T}=6.53$; cluster level pFWE $<0.004)$, and without $(34,-42,52$; $\mathrm{T}=6.59$; cluster level $\mathrm{pFWE}<0.003)$ adding age as a nuisance variable (Figure 5). Table 2 shows differences between groups at a $p<0.001$ uncorrected threshold for positive and negative contrasts.

\section{Discussion}

The main finding of this cross-sectional study is that, unlike healthy subjects, migraine patients exhibit brain metabolic modifications in relation with the ageing process during the ages where migraine prevalence peaks. These modifications are localized in regions that have been linked to the pathophysiology of the disease on numerous occasions in the past. Furthermore, in migraineurs, age is more robustly correlated with metabolic changes than disease duration or the number of monthly migraine days. Put altogether, results of this study suggest that from a metabolic perspective, more than the strain produced by intermittent headache attacks, the physiological state of the brain of migraine patients is modelled by the constant interaction with the environment. 


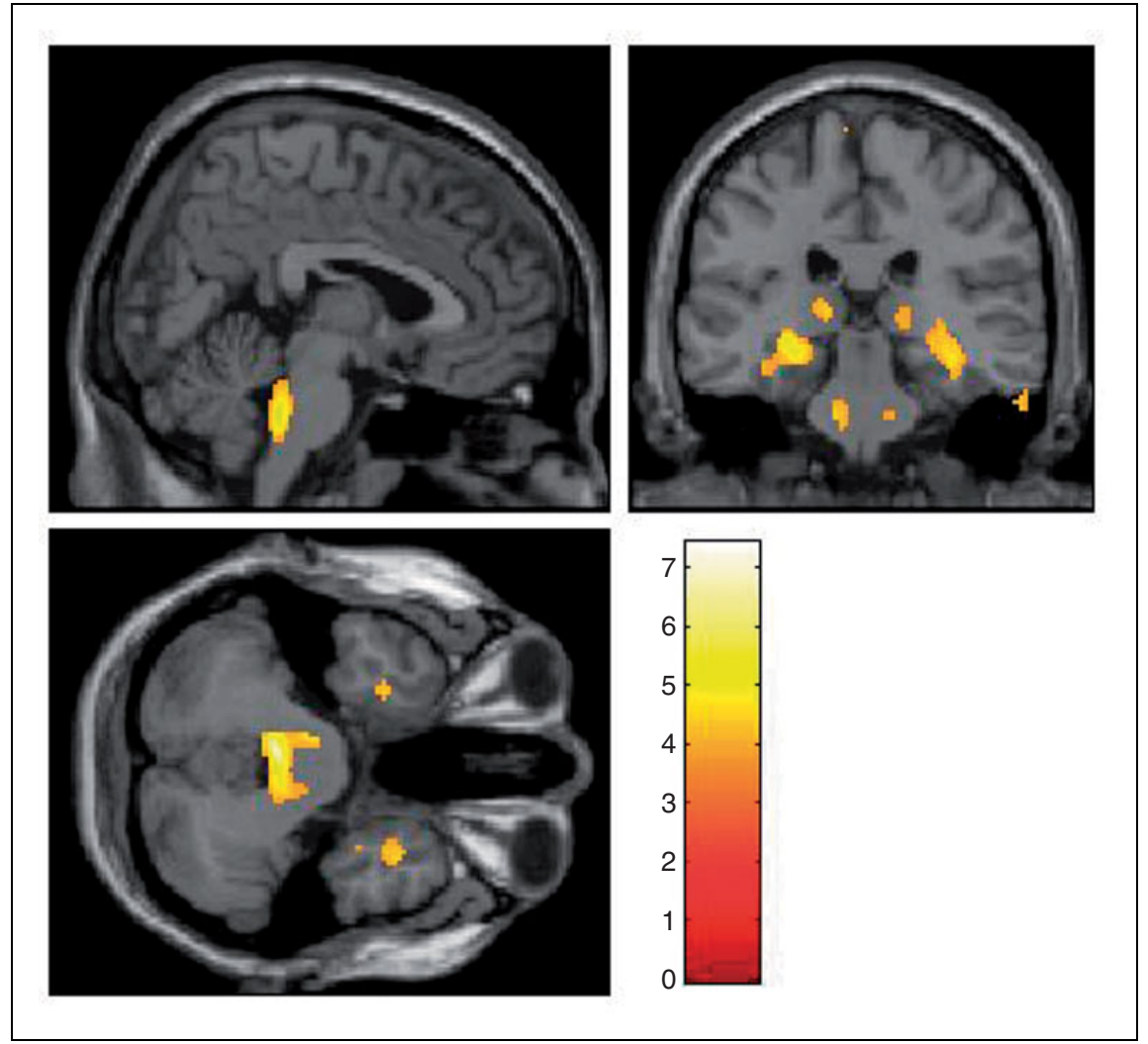

Figure 2. $\mathrm{t}$-values (color-coded) of voxels where ${ }^{18}$ FDG uptake is postively correlated with age in 19 interictal episodic migraine without aura patients $(4,-28,-36)$. See color scale on the bottom right. Regions include the brainstem, and two large, almost symmetrical, bilateral, clusters extending over the hippocampus, the parahippocampal and fusiform gyri, and some voxels belonging to the thalamus.

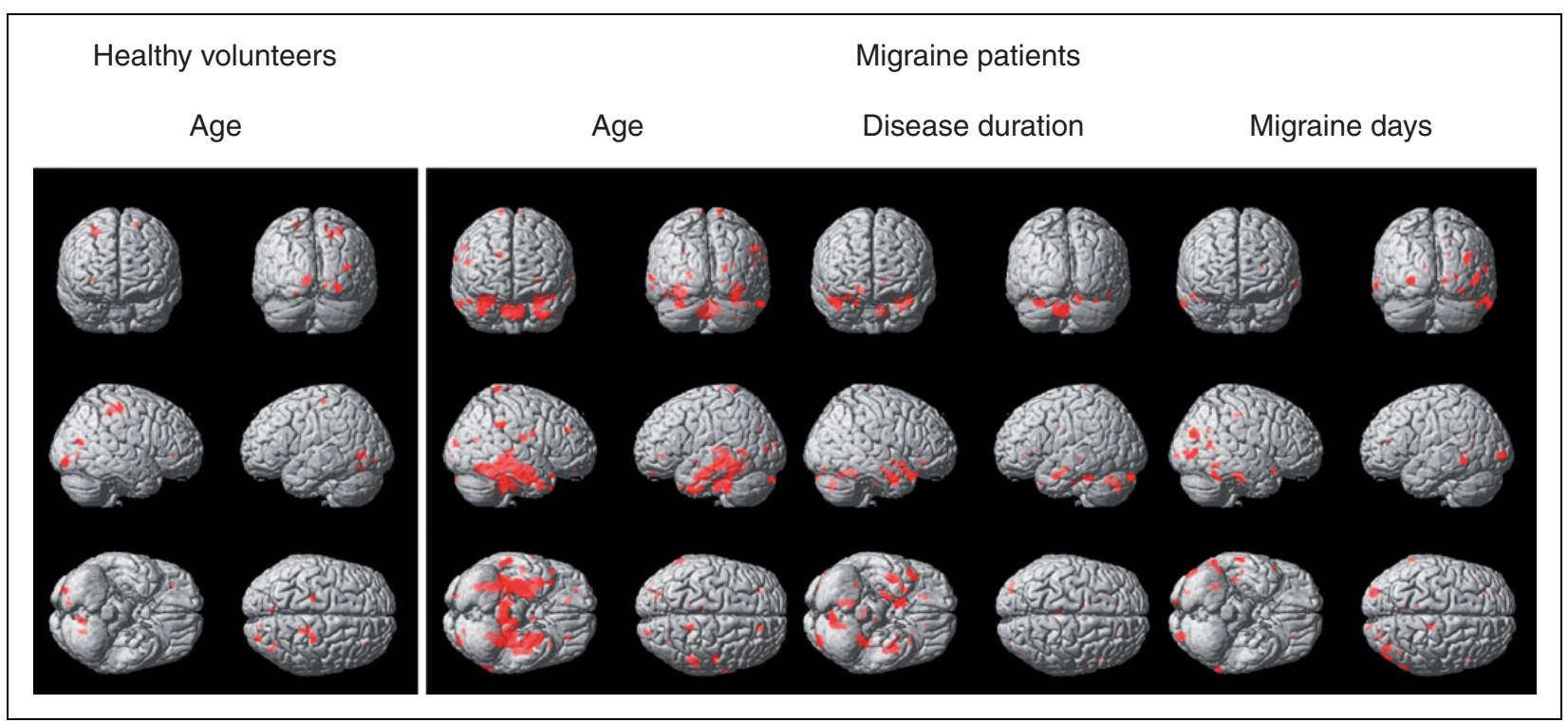

Figure 3. Positive correlations (color-coded) between ${ }^{18} \mathrm{FDG}$ uptake and the different clinical parameters in healthy volunteers and migraine patients at a $p<0.00$ I uncorrected threshold. 


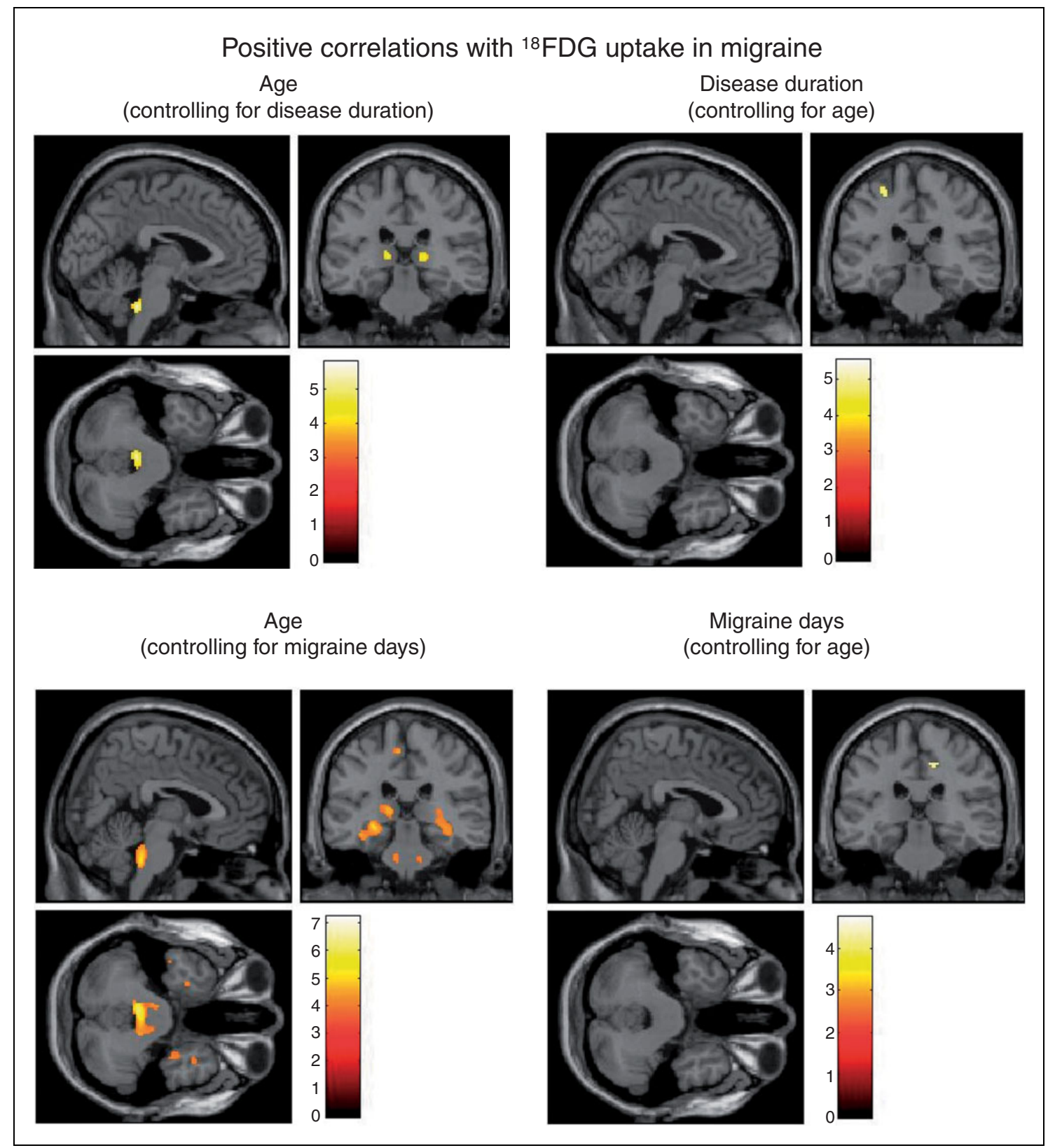

Figure 4. Correlations between ${ }^{18} \mathrm{FDG}$ uptake and age (controlling for disease duration (upper left) or migraine days (bottom left)), and FDG-uptake and disease duration (controlling for age (top right)) or migraine days (controlling for age (bottom right)) at a $p<0.00$ I uncorrected threshold. Voxels in the posterior pons, parahippocampal gyrus, fusiform gyrus, and thalamus where ${ }^{18} \mathrm{FDG}$ uptake is postively correlated with age in migraine patients still appear after controlling for the other variables.

More than 10 years ago, the first study addressing the correlation between FDG uptake and clinical variables in migraine patients was published. Focusing on the imprint of the disease on the brain, age was considered a nuisance variable in the analyses (12). Results showed a progressive glucose hypometabolism in relation to disease duration in brain regions belonging to the central pain matrix. Positive correlations were only found in white matter regions (frontal and parietal), and no changes were observed in the brainstem (12). Our contrasting results clearly challenge the consideration of age as a covariate of no interest and place it in a rather central position in the model.

\section{The posterior pons, age, and migraine}

The limited spatial resolution of PET makes a precise, unequivocal, anatomical segregation almost impossible, 


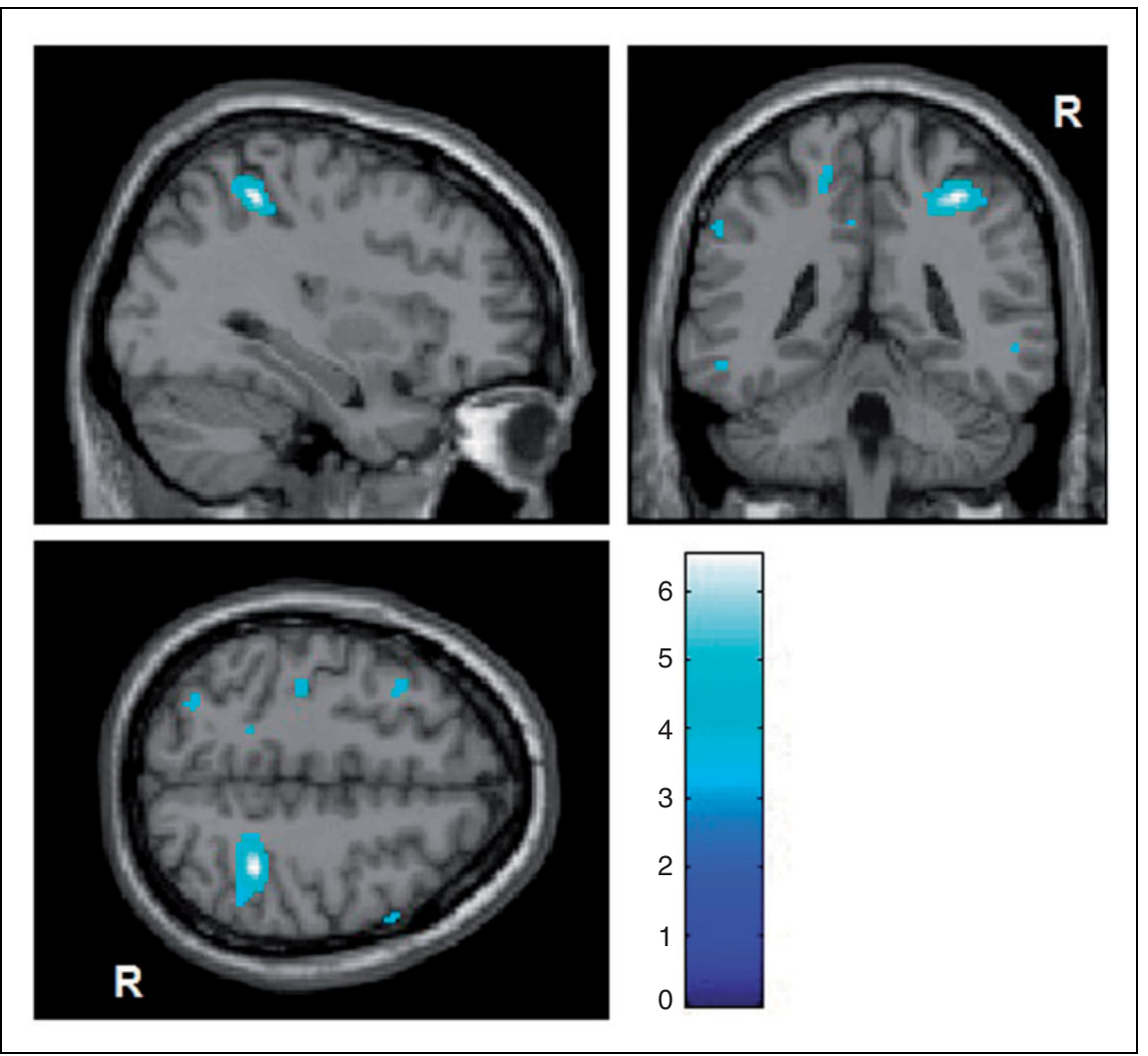

Figure 5. Cluster of significantly reduced ${ }^{18} \mathrm{FDG}$ uptake in the somatosensory cortex of migraine patients with respect to healthy controls. The letter $\mathrm{R}$ indicates the right side of the image.

and especially at the brainstem level. The voxels in the pons where FDG-uptake showed a positive correlation with age in migraine patients in our study could involve numerous structures like the trigeminal brainstem nuclei, the dorsal raphe nucleus, the nucleus tractus solitarius, the locus coeruleus and the nucleus cuneatus, to name a few (13). Therefore, although a precise anatomo-functional interpretation of our findings would remain largely hypothetical, mechanistic assumptions about the pathophysiology of the disease could readily be drawn. The posterior pons has been repeatedly associated with migraine in the past, up to the point of being considered a migraine generator (14-16). Activation of this region has been described during both the premonitory and the headache phases of the disease (17), suggesting that it might occur as a protective mechanism, aiming to refrain from an imminent headache attack. If this is the case, the possibility arises that age-related increases in metabolism in the posterior pons might represent a long-term compensatory effort trying to impede headache crises from occurring. Such a hypothesis would serve as a justification for the common clinical observation of migraine disappearing (or reducing in severity and frequency) with advancing age. If the metabolism (and then activity) of the brainstem increases with age in migraine patients, it would forcefully enhance thalamocortical activity, and finally lead to a normalization of the otherwise increased cortical responsivity (18). Metabolic connectivity analyses coupled with electrophysiological studies would be needed in order to verify this hypothesis. Furthermore, if metabolic modifications in the brainstem of migraine patients are indeed the explanation behind migraine waning with age, such an adaptive change could be externally facilitated in order to control migraine symptoms. As before, this possibility would also need to be corroborated in future experiments.

Just as mentioned in the introduction, our study is not the first to describe brain age-related modifications in migraine patients. Chong et al. evaluated the differences in cortical thickness in relation with age between migraineurs and healthy controls (10). They reported atypical cortical thinning in association with age in migraine, which affected the postcentral gyri bilaterally, as well as the right fusiform gyrus and right temporal pole. The authors concluded that migraine patients present age-related cortical thinning that occurs regardless of disease duration and attack frequency (10). Even if the strongest correlation we observed was located at the 
Table 2. Differences in brain metabolism in patients with migraine compared with healthy controls at a $p<0.00 \mathrm{I}$ (uncorrected) threshold. The region of significantly different metabolism at a pFWE $<0.05$ statistical threshold is shaded in grey.

\begin{tabular}{|c|c|c|c|c|c|c|c|}
\hline & $x, y, z\{m m\}$ & $\mathrm{x}, \mathrm{y}, \mathrm{z}\{\mathrm{mm}\}$ & $x, y, z\{m m\}$ & $\begin{array}{l}\text { Cluster size } \\
\text { (Voxels) }\end{array}$ & $\begin{array}{l}\text { Peak } \\
\mathrm{T}\end{array}$ & $\begin{array}{l}\text { Peak } \\
\text { P(FWE-corr) }\end{array}$ & $\begin{array}{l}\text { Peak } \\
\text { P(unc) }\end{array}$ \\
\hline Right superior parietal lobe & 34 & -42 & 52 & 261 & 6.5884 & 0.0031 & 0.0000 \\
\hline Left inferior frontal lobe & -48 & 24 & 14 & 67 & 4.7576 & 0.3085 & 0.0000 \\
\hline \multirow[t]{2}{*}{ Left middle temporal gyrus } & -64 & -54 & 2 & 90 & 4.6634 & 0.3722 & 0.0000 \\
\hline & -60 & -48 & 10 & & 3.7103 & 0.9851 & 0.0003 \\
\hline \multirow[t]{2}{*}{ Left precuneus } & -12 & -56 & 58 & 161 & 4.6215 & 0.4030 & 0.0000 \\
\hline & -16 & -42 & 58 & & 4. 1048 & 0.8276 & 0.0001 \\
\hline Left cuneus & -8 & -76 & 18 & 61 & 4.5916 & 0.4259 & 0.0000 \\
\hline Left precentral gyrus & -36 & -24 & 58 & 52 & 4.4875 & 0.5104 & 0.0000 \\
\hline Right Rolandic operculum & 54 & 10 & -2 & 64 & 4.1814 & 0.7708 & 0.0001 \\
\hline Left superior occipital gyrus & -20 & -78 & 30 & 56 & 4.1624 & 0.7856 & 0.0001 \\
\hline Right parahipocampal gyrus & 22 & -24 & -24 & 30 & 4.1226 & 0.8150 & 0.0001 \\
\hline Left frontal infero-orbital gyrus & -40 & 30 & -4 & 74 & 4.0980 & 0.8322 & 0.0001 \\
\hline Left supramarginal gyrus & -62 & -30 & 32 & 30 & 4.0971 & 0.8329 & 0.0001 \\
\hline Left prefrontal cortex & -16 & 42 & 26 & 25 & 4.0368 & 0.8716 & 0.0001 \\
\hline Left parahippocampus & -16 & -26 & -22 & 29 & 4.0127 & 0.8855 & 0.0001 \\
\hline Left angular gyrus & -56 & -44 & 40 & 14 & 3.9992 & 0.8929 & 0.0001 \\
\hline Right prefrontal cortex & 54 & 10 & 48 & 29 & 3.9495 & 0.9177 & 0.0002 \\
\hline Left parietal white matter & -16 & -54 & 32 & 13 & 3.8687 & 0.9495 & 0.0002 \\
\hline Left superior parietal gyrus & -34 & -50 & 46 & 26 & 3.8507 & 0.9552 & 0.0002 \\
\hline Left pre-motor cortex & -52 & -2 & 36 & 26 & 3.7889 & $0.97 \mid 4$ & 0.0003 \\
\hline Right frontal infero-orbital gyrus & 42 & 34 & 0 & 24 & 3.7559 & 0.9780 & 0.0003 \\
\hline Left frontal white matter & -32 & 12 & 48 & 18 & 3.7546 & 0.9782 & 0.0003 \\
\hline Left prefrontal cortex & -32 & 34 & 30 & 8 & 3.7333 & 0.9818 & 0.0003 \\
\hline Left extra-striate visual cortex & -36 & -72 & -12 & 15 & 3.7329 & 0.9818 & 0.0003 \\
\hline Left extra-striate visual cortex & -46 & -80 & 0 & 15 & 3.6834 & 0.9884 & 0.0004 \\
\hline Right middle temporal gyrus & 64 & -6 & -24 & 4 & 3.6652 & 0.9902 & 0.0004 \\
\hline Right supramarginal gyrus & 64 & -26 & 30 & 3 & 3.6223 & 0.9937 & 0.0004 \\
\hline Left superior parietal lobe & -28 & -66 & 50 & 15 & 3.6012 & 0.9950 & 0.0005 \\
\hline Right superior parietal lobe & 24 & -52 & 64 & 17 & 3.5886 & 0.9956 & 0.0005 \\
\hline Left fusiform gyrus & -56 & -42 & -12 & 6 & 3.5533 & $0.997 \mid$ & 0.0005 \\
\hline Left fusiform gyrus & -42 & -46 & -24 & 5 & $3.547 I$ & 0.9973 & 0.0005 \\
\hline Right frontal white matter & 16 & -4 & 58 & 4 & 3.5364 & 0.9976 & 0.0006 \\
\hline Right pre-motor cortex & 56 & 2 & 36 & 3 & 3.5146 & 0.9982 & 0.0006 \\
\hline Left superior parietal lobe & -22 & -74 & 42 & 4 & 3.4428 & 0.9993 & 0.0007 \\
\hline Right pre-frontal cortex & 16 & 34 & 38 & 4 & 3.4154 & 0.9996 & 0.0008 \\
\hline Left dorsal posterior cingulate & -6 & -42 & 42 & 2 & 3.4090 & 0.9996 & 0.0008 \\
\hline Left supramarginal gyrus & -58 & -20 & 36 & 3 & 3.4086 & 0.9996 & 0.0008 \\
\hline Left middle temporal gyrus & 56 & -42 & -6 & I & 3.3640 & 0.9998 & 0.0009 \\
\hline
\end{tabular}

Hypermetabolism in migraine

\begin{tabular}{lccccccc}
\hline & & & & Cluster & Peak & $\begin{array}{c}\text { Peak } \\
\text { Eeak } \\
\end{array}$ \\
& $\mathbf{x , y , z}\{\mathrm{mm}\}$ & $\mathbf{x , y , z}\{\mathrm{mm}\}$ & $\mathbf{x , y , z}\{\mathrm{mm}\}$ & Equivk & $\mathrm{T}$ & $\mathrm{P}(\mathrm{FWE}-\mathrm{corr})$ & $\mathrm{P}(\mathrm{unc})$ \\
\hline Right angular + supramarginal gyri & 34 & -50 & 34 & 53 & 4.4652 & 0.5294 \\
Right middle frontal gyrus & 38 & 4 & 38 & 36 & 3.8750 & 0.9474 & 0.0000 \\
\hline
\end{tabular}


brainstem, a region that evidently cannot be compared with the study by Chong et al. analysing the cerebral cortex, the correlated regions that we found at the cortical level do not strictly overlap with the ones they described. One possible explanation for the contrasting results between our study and the one by Chong et al. would be that cortical thinning in migraine patients is not paired with noticeable metabolic modifications. Studies simultaneously addressing both variables are warranted. Of note, the strongest negative correlation we observed in the cortex, located at the right temporoparietal junction (a key region of sensory processing likely involved in migraine $(14,18-22)$, did not reach statistical significance after correcting for multiple comparisons.

\section{Differences with normal ageing}

In healthy subjects, the normal process of ageing induces a progressive hypometabolism in several brain areas. These areas include the anterior cingulate, orbitofrontal, dorsolateral and medial prefrontal cortices in the frontal lobe, the supramarginal, superior and inferior parietal cortices in the parietal lobe, the lateral temporal cortex and temporal poles in the temporal lobe, and the insula. Least affected regions during the ageing process are the primary motor and occipital cortices, precuneus, and notably the mesial temporal lobes and thalamus $(23,24)$. The distribution of this hypometabolism in normal aging inversely follows the development line of the human brain (i.e. structures that first developed are spared from an age-related metabolic deterioration whereas the frontal regions, which become metabolically active during later stages of the central nervous system development, are the most consistently affected by aging) $(25,26)$. In this study no significant metabolic modifications in relation to age were observed in the healthy subjects group. This is likely due to the younger range of age of our control sample (21-59 years) with respect to cohorts in most studies addressing this phenomenon (i.e. 20-70 years). However, and perhaps more surprising, is that in migraine patients metabolism in the mesial temporal lobe and thalamus was not only maintained but rather progressively increased. Preservation of metabolism in these structures is consistent with the functional importance of thalamo-limbic circuits in sensory integration, arousal, emotion, and memory $(25,26)$, and it is likely that these same underpinnings push it forth in migraine.

\section{Between-groups comparison}

In our study, the strongest difference observed when comparing migraine patients and healthy volunteers, both with or without controlling for the effect of age, was a region of reduced FDG uptake in migraineurs situated in the right post-central gyrus. Several ${ }^{18}$ FDG PET studies have compared migraine patients and healthy controls in the past $(12,27,28)$. In one study with a smaller sample size compared to the present work, an increased metabolism in the posterior white matter of migraine patients was reported. The minimal extent threshold of 500 voxels that the authors employed could have discarded any possible clusters situated at the brainstem level (27). A couple of years later, in a larger study, Kim et al. found a significant hypometabolism in the primary somatosensory cortex similar to the one we report in the present study. They also described a reduced metabolism in other regions belonging to the pain matrix (i.e. the insula, cingulate cortex, and prefrontal cortex). Except for the anterior cingulate cortex, results from our exploratory analyses performed at a more permissive threshold $(p<0.001$ uncorrected) exhibit some degree of overlapping with their results (see Table 2). Finally, in one study including chronic migraine patients, Aurora et al. have described a relatively increased metabolism in the pons of migraineurs (28).

\section{Limitations}

The major limitation of our study is that PET spatial resolution is limited, and thus a precise, unambiguous identification of small regions cannot be achieved through this method. Also, age and disease duration are correlated in our sample of patients $(r=0.698$; $p<0.01)$, and thus the particular effect of each cannot be entirely separated. Furthermore, a positive control (e.g. chronic pain) is missing in this study and because of that the findings herein reported cannot be proven to be migraine specific. The relatively small number of subjects in each group could also be considered a limitation of the present study. In addition, although not considered in the original study design, on the basis of our findings it seems plausible that performing tests to evaluate cognitive and other cerebral functions may have ameliorated our comprehension of the outcomes. Finally, recall bias could certainly reduce precision when estimating disease duration. Therefore, although encouraging, our results would need to be corroborated in a longitudinal study specifically addressing the cerebral metabolic modifications throughout the life span in migraine patients.

\section{Conclusions}

Migraine patients exhibit metabolic modifications in the brain in relation with ageing. As such, age 
is correlated with metabolic changes in key regions of the brain associated with migraine's pathophysiology to a better extent than disease duration or the number of migraine days. More than the repeated headache attacks, an adaptive response to constant exposure to environmental stimuli seemingly models the brain of migraine sufferers.

\section{Key findings}

- Episodic migraine patients exhibit specific metabolic brain modifications while ageing.

- Age is correlated with metabolic changes in key regions of the brain previously associated with migraine's pathophysiology to a better extent than disease duration or the number of monthly migraine days.

\section{Acknowledgements}

The contribution of the IRCCS - Fondazione Bietti, Research Unit of Neurophysiology of Vision and Neurophthalmology, in this paper was supported by the Italian Ministry of Health and Fondazione Roma. The authors thankfully acknowledge Professor Roland Hustinx, Chief of the Nuclear Medicine Service at the Centre Hospitalier Universitaire de Liège, for his collaboration on this study, and Professor Lars Jacob Stovner, from the Department of Neuroscience, Norwegian University of Science and Technology, for his valuable help in the construction of Figure 1.

\section{Declaration of conflicting interests}

The authors declared no potential conflicts of interest with respect to the research, authorship, and/or publication of this article.

\section{Funding}

The authors disclosed receipt of the following financial support for the research, authorship, and/or publication of this article: This project forms part of the EUROHEADPAIN project - FP7 n ${ }^{\circ} 602633$. The work of Gianluca Coppola and Vincenzo Parisi is supported by the IRCCS Fondazione Bietti.

\section{ORCID iD}

Marco Lisicki (D) http://orcid.org/0000-0001-9765-6759 Gianluca Coppola (D) http://orcid.org/0000-0002-8510-6925 Delphine Magis (D) http://orcid.org/0000-0002-4392-9413

\section{References}

1. Jensen R and Stovner LJ. Epidemiology and comorbidity of headache. Lancet Neurol 2008; 7: 354-361.

2. Steiner TJ, Stovner LJ, Vos T, et al. Migraine is first cause of disability in under 50s: Will health politicians now take notice? J Headache Pain 2018; 19: 17. DOI: 10.1186/ s10194-018-0846-2.

3. Linde M, Gustavsson A, Stovner LJ, et al. The cost of headache disorders in Europe: The Eurolight project. Eur J Neurol 2012; 19: 703-711.

4. Borsook D, Maleki N, Becerra L, et al. Understanding migraine through the lens of maladaptive stress responses:
A model disease of allostatic load. Neuron 2012; 73 : 219-234.

5. Goadsby PJ, Holland PR, Martins-Oliveira M, et al. Pathophysiology of migraine: A disorder of sensory processing. Physiol Rev 2017; 97: 553-622.

6. Bigal ME and Lipton RB. The epidemiology, burden, and comorbidities of migraine. Neurol Clin 2009; 27: 321-334.

7. Stovner LJ, Zwart JA, Hagen K, et al. Epidemiology of headache in Europe. Eur $J$ Neurol 2006; 13: 333-345.

8. Lagman-Bartolome AM and Lay C. Pediatric migraine variants: A review of epidemiology, Diagnosis, treatment, and outcome. Curr Neurol Neurosci Rep 2015; 15: 34. DOI: $10.1007 / \mathrm{s} 11910-015-0551-3$.

9. Ripa P, Ornello R, Degan D, et al. Migraine in menopausal women: A systematic review. Int J Womens Health 2015; 7: 773-782.

10. Chong CD, Dodick DW, Schlaggar BL, et al. Atypical age-related cortical thinning in episodic migraine. Cephalalgia 2014; 34: 1115-1124.

11. Headache Classification Committee of the International Headache Society (IHS). The International Classification of Headache Disorders, 3rd edition (beta version). Cephalalgia 2013; 53: 137-146.

12. Kim JH, Kim S, Suh SI, et al. Interictal metabolic changes in episodic migraine: A voxel-based FDG-PET study. Cephalalgia 2010; 30: 53-61.

13. Vila-Pueyo M, Hoffmann J, Romero-Reyes M, et al. Brain structure and function related to headache: Brainstem structure and function in headache. Cephalalgia. Epub ahead of print 1 January 2018. DOI: 10.1177/0333102418784698.

14. Weiller C, May A, Limmroth V, et al. Brain stem activation in spontaneous human migraine attacks. Nat Med 1995; 1: 658-660.

15. Denuelle M, Fabre N, Payoux P, et al. Hypothalamic activation in spontaneous migraine attacks. Headache 2007; 47: 1418-1426.

16. Stankewitz A, Aderjan D, Eippert F, et al. Trigeminal nociceptive transmission in migraineurs predicts migraine attacks. J Neurosci 2011; 31: 1937-1943.

17. Maniyar FH, Sprenger T, Monteith T, et al. Brain activations in the premonitory phase of nitroglycerin-triggered migraine attacks. Brain 2014; 137: 232-241. 
18. Lisicki M, D’Ostilio K, Coppola G, et al. Brain correlates of single trial visual evoked potentials in migraine: More than meets the eye. Front Neurol 2018; 9: 393. DOI: 10.3389 /fneur.2018.00393.

19. Mickleborough MJS, Ekstrand C, Gould L, et al. Attentional network differences between migraineurs and nonmigraine controls: fMRI evidence. Brain Topogr 2016; 29: 419-428.

20. Demarquay G, Royet J, Mick G, et al. Olfactory hypersensitivity in migraineurs: A $\mathrm{H}_{2}{ }^{15} \mathrm{O}$-PET Study. Cephalalgia 2008; 28: 1069-1080.

21. Hougaard A, Amin FM, Hoffmann MB, et al. Interhemispheric differences of fMRI responses to visual stimuli in patients with side-fixed migraine aura. Hum Brain Mapp 2014; 35: 2714-2723.

22. Lisicki M, D’Ostilio K, Coppola G, et al. Increased functional connectivity between the right temporo-parietal junction and the temporal poles in migraine without aura. Cephalalgia Reports 2018; 1: 1-4. DOI: 10.1177/ 2515816318804823.

23. Berti V, Mosconi L and Pupi A. Brain: Normal variations and benign findings in fluorodeoxyglucose-PET/ computed tomography imaging. PET Clin 2014; 9: $129-140$.
24. Bonte $\mathrm{S}$, Vandemaele $\mathrm{P}$, Verleden $\mathrm{S}$, et al. Healthy brain ageing assessed with 18F-FDG PET and age-dependent recovery factors after partial volume effect correction. Eur J Nucl Med Mol Imaging 2017; 44: 838-849.

25. Grieve SM, Clark CR, Williams LM, et al. Preservation of limbic and paralimbic structures in aging. Hum Brain Mapp 2005; 25: 391-401.

26. Kalpouzos G, Chételat G, Baron JC, et al. Voxel-based mapping of brain gray matter volume and glucose metabolism profiles in normal aging. Neurobiol Aging 2009; 30: $112-124$.

27. Kassab M, Bakhtar O, Wack D, et al. Resting brain glucose uptake in headache-free migraineurs. Headache 2009; 49: 90-97.

28. Aurora SK, Barrodale PM, Tipton RL, et al. Brainstem dysfunction in chronic migraine as evidenced by neurophysiological and positron emission tomography studies. Headache 2007; 47: 996-1003.

29. Lipton RB, Bigal ME, Diamond M, et al. Migraine prevalence, disease burden, and the need for preventive therapy. Neurology 2007; 68: 343-349. 